\title{
ENFERMAGEM NA PREVENÇÃO E CONTROLE DE INFECÇÃO DE NEONATOS: REVISÃO INTEGRATIVA
}

\author{
Nursing in the prevention and control of infectionl of neonates: integrative review
}

Caroline de Andrade Bezerra ${ }^{1}$ (i), Thaís Kelly Caetano Martinss ${ }^{-1}$, Carla Andréia Santos Silva ${ }^{-1}$, Pedro Henrique da Silva Ramos $^{2}$ (i), Roberto Nascimento de Albuquerque ${ }^{3 *}$ (D)

\section{RESUMO}

Mundialmente, o número de mortes entre neonatos foi de 2,4 milhões em 2019, ocorrendo aproximadamente 7.000 mortes por dia. Neste mesmo ano, o Brasil apresentou um registro de 24.504 óbitos de neonatos por causas evitáveis. Os enfermeiros desempenham importantes ações no cuidado de neonatos nascidos em qualquer condição gestacional, sejam eles saudáveis ou com infecção congênita ou adquirida. Nesse contexto, o objetivo deste estudo foi identificar ações de saúde desenvolvidas pela equipe de enfermagem na prevenção e controle de infecção de neonatos. Tratou-se de uma revisão integrativa de literatura nas bases de dados eletrônicas LILACS, BDENF e MEDLINE, com artigos publicados entre 2011 a 2021, disponíveis na íntegra, em português, inglês e espanhol. Inicialmente foram encontrados 386. Após a aplicação dos critérios de inclusão e exclusão, foram selecionados 15 artigos para comporem a discussão deste artigo. Importantes ações da equipe de enfermagem foram demonstradas, destacando-se a lavagem das mãos, a utilização de proteção pessoal, os cuidados com a pele e para a implantação de cateteres centrais, orientações relacionadas à amamentação e os cuidados envolvendo a participação dos pais. Conclui-se que os enfermeiros neonatais realizam intervenções de alta complexidade, além de atuar na prevenção e promoção à saúde. Trabalhos educativos continuados para seu aperfeiçoamento é importante visando atualizar e melhorar a qualidade e segurança do cuidado de enfermagem.

Palavras-chave: Controle de Infecções; Recém-Nascido; Cuidados de enfermagem;

\section{ABSTRACT}

Worldwide, the number of deaths among newborns was 2.4 million in 2019, with approximately 7,000 deaths occurring per day. In that same year, Brazil presented a record of 24,504 deaths of newborns from preventable causes. Nurses perform important actions in the care of newborns born in any gestational condition, whether they are healthy or with congenital or acquired infection. In this context, the objective of this study was to identify health actions developed by the nursing team in the prevention and control of infection in newborns. This was an integrative literature review in LILACS, BDENF and MEDLINE electronic databases, with articles published between 2011 and 2021, available in full, in Portuguese, English and Spanish. Initially, 386 were found. After applying the inclusion and exclusion criteria, 15 articles were selected to compose the discussion of this article. Important actions of the nursing team were demonstrated, highlighting hand washing, the use of personal protection, skin care and the implantation of central catheters, guidelines related to breastfeeding and care involving the participation of parents. It is concluded that neonatal nurses perform highly complex interventions, in addition to acting in prevention and health promotion. Continued educational work for its improvement is important in order to update and improve the quality and safety of nursing care.

Keywords: Infection control; Newborn; Nursing Care.

1. Graduada em Ciências Biológicas. Mestre em Imunologia. Doutora em Ciências Genômicas e Biotecnologia. Graduanda em Enfermagem pelo Centro Universitário de Brasília (CEUB), Brasília, DF, Brasil

2. Graduanda em Enfermagem pelo Centro Universitário de Brasíllia (CEUB), Brasília, DF, Brasil.

3. Enfermeiro. Mestre e Doutor em Enfermagem pela Universidade de Brasília (UnB). Professor Titular do Centro Universitário de Brasília (CEUB), Brasília, DF, Brasil.

*Autor para Correspondência. E-mail: roberto.albuquerque@ ceub.edu.br 


\section{INTRODUÇÃO}

Na esfera global, a Organização Mundial da Saúde (OMS) relatou que 2,4 milhões de neonatos morreram em 2019, ocorrendo aproximadamente 7.000 mortes por dia. Este levantamento de dados mostra um decréscimo no número de óbitos neonatais quando comparado com os 5,0 milhões em 1990. Países de média e baixa renda apresentaram as maiores taxas de mortalidade neonatal. Os óbitos nos primeiros 28 dias após o nascimento estão associados a falta de atendimento e tratamento qualificados durante o parto, imediatamente após o nascimento e nos primeiros dias de vida, sendo as principais causas na maioria das mortes os partos prematuros, complicações relacionadas ao parto como asfixia no parto ou a falta de respiração, defeitos congênitos e infecções neonatais ${ }^{1}$.

Segundo dados brasileiros, o país apresentou um registro de 24.504 óbitos de neonatos por causas evitáveis em $2019^{2}$. Neste contexto, com a finalidade de sistematizar a vigilância das infecções relacionadas à assistência à saúde (Iras) em neonatologia, a Agência Nacional de Vigilância Sanitária (ANVISA) desenvolveu um manual de Definição dos Critérios Nacionais de Infecções Relacionadas à Assistência à Saúde. Este documento trata-se de uma ferramenta para estabelecer estratégias de prevenção e controle das infecções em neonatos, visando uma ação mais abrangente das infecções do período pré-natal, perinatal e neonatal ${ }^{3}$.

Trabalhos conjuntos entre OMS, Ministérios da Saúde e parceiros têm sido realizados para o avanço dos serviços de saúde qualificados e acessíveis para neonatos, inclusive por meio do fortalecimento da enfermagem neonatal ${ }^{1}$. Os profissionais de enfermagem neonatal atuam na arte e na ciência do cuidado de neonatos nascidos em qualquer condição gestacional, sejam eles saudáveis ou com infecção congênita ou adquirida. Os enfermeiros neonatais trabalham junto a equipes multiprofissionais, além de participar de pesquisas e implementar resultados de estudos científicos nessa área. Além disso, também estão comprometidos na aprendizagem profissional continuada, fornecendo educação e liderando esforços de melhoria da qualidade e eficácia de suas práticas ${ }^{4}$.

Dentre a diversidade de competências dos enfermeiros, diferentes estudos científicos têm demonstrado o importante papel destes profissionais na prevenção e controle de infecção de neonatos ${ }^{5-8}$.

Neste contexto, o presente estudo teve como objetivo identificar ações de saúde desenvolvidas pela equipe de enfermagem na prevenção e controle de infecção de neonatos.

\section{METODOLOGIA}

Este estudo caracteriza-se como uma revisão integrativa de literatura a qual proporciona uma visão geral dos conhecimentos existentes em uma área específica, além de possibilitar a identificação de lacunas na base do conhecimento a serem preenchidas por novos estudos ${ }^{9}$.

A elaboração dessa revisão compreendeu o seguimento de seis etapas: (1) formulação da questão para nortear a pesquisa; (2) definição de estratégias de busca, determinando os descritores e as bases de dados; (3) definição dos critérios de inclusão e exclusão; (4) identificação e seleção dos estudos; (5) análise das informações dos estudos selecionados; e (6) divulgação dos resultados e discussão.

A questão elaborada para nortear o desenvolvimento deste estudo foi: "Quais as ações desenvolvidas pela equipe de enfermagem previnem e controlam as infecções de neonatos? ". Os descritores estabelecidos para busca de publicações nas bases de dados foram "enfermagem", "prevenção e controle de infecções" e "neonato", sendo os três utilizados juntos por meio do operador booleano "AND". As consultas foram realizadas em março de 2021 na base de dados eletrônica Biblioteca Virtual em Saúde (BVS).

Como critérios de inclusão, foram selecionados artigos nas bases de dados Literatura Latino-Americana e do Caribe em Ciências da Saúde (LILACS), Biblioteca Virtual de Enfermagem (BDENF) e Medical Literature Analysis and Retrieval System Online (MEDLINE). Os critérios de inclusão foram: artigos disponíveis na íntegra nos idiomas português, inglês e espanhol e publicados nos últimos 10 anos. Os critérios de exclusão foram artigos que não respondiam à questão norteadora, revisões sistemáticas ou integrativas da literatura, cartas ao leitor, editoriais, teses, dissertações, livros, capítulos de livros e matérias de jornal.

\section{RESULTADOS}

Inicialmente foram localizadas 386 publicações. Com a aplicação dos critérios de inclusão, houve uma restrição para 86 artigos, sendo 13 indexados na LILACS, 11 na BDENF e 71 na MEDLINE, dentre os idiomas português, inglês e espanhol no período de 2011 a 2021. Os títulos e os resumos destes artigos foram avaliados quanto à conformidade com a temática do estudo, ocorrendo a seleção de 34 publicações e 52 exclusões. Em seguida, procedeu-se à eliminação de 8 artigos por duplicidade e mais 8 pela aplicação dos critérios de exclusão, assim permanecendo 18 trabalhos para a leitura completa. Após a avaliação na íntegra das 18 publicações, 3 foram excluídas por não apresentarem um conteúdo pertinente que demonstre ações do profissional 
de enfermagem para prevenção e controle de infecção neonatais, restando 15 trabalhos para constituir o banco de dados dessa pesquisa.

O caminho metodológico para o corpus da discussão desta pesquisa encontra-se na Figura 1.

Figura 1 - Diagrama de fluxo do processo de seleção de artigos da revisão integrativa.

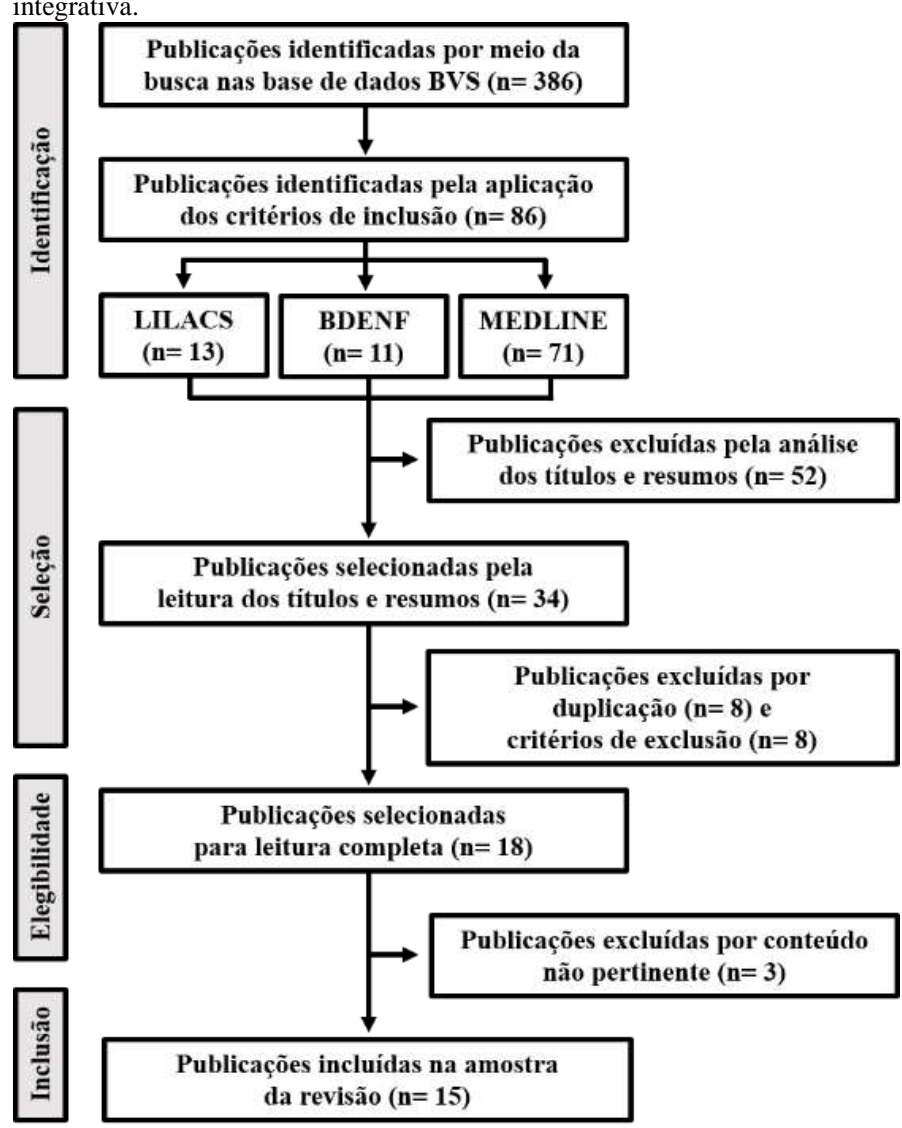

Fonte: os autores.

Os 15 artigos selecionados estão sistematizados no Quadro 1, conforme autores, ano e periódico de publicação, título e objetivos dos estudos.

Quadro 1. Descrição dos estudos selecionados em bases de dados, segundo autores, ano, periódicos, título, objetivo e tipo do estudo.

\begin{tabular}{|l|l|l|}
\hline \multicolumn{1}{|c|}{$\begin{array}{l}\text { Autores e ano / } \\
\text { periódicos }\end{array}$} & \multicolumn{1}{|c|}{ Título } & \multicolumn{1}{c|}{ Objetivos } \\
\hline $\begin{array}{l}\text { Cunha et al., } \\
2013\end{array}$ & $\begin{array}{l}\text { Representações sociais } \\
\text { de infecção neonatal } \\
\text { elaboradas por } \\
\text { enfermeiras }\end{array}$ & $\begin{array}{l}\text { Apreender as representações } \\
\text { sociais da infecção neonatal } \\
\text { elaboradas por enfermeiras e } \\
\text { analisar a relação dessas } \\
\text { representações com a } \\
\text { assistência ao recém-nascido } \\
\text { com infecção. }\end{array}$ \\
$\begin{array}{l}\text { Enfermagem } \\
\text { UERJ }\end{array}$ & $\begin{array}{l}\text { Improvement of } \\
\text { adherence to hand } \\
\text { hygiene practice using } \\
\text { a multimodal } \\
\text { intervention program } \\
\text { in a neonatal intensive } \\
\text { care }\end{array}$ & $\begin{array}{l}\text { Avaliar o efeito da } \\
\text { implementação de um } \\
\text { programa de intervenção } \\
\text { multimodal para melhoria das } \\
\text { práticas de higiene das mãos } \\
\text { na adesão às diretrizes de } \\
\text { higiene entre todos os }\end{array}$ \\
\hline $\begin{array}{l}\text { Journal of } \\
\text { nursing care } \\
\text { quality }\end{array}$ & \multicolumn{2}{|c}{} \\
\hline
\end{tabular}

\begin{tabular}{|c|c|c|}
\hline & & $\begin{array}{l}\text { profissionais de saúde da } \\
\text { UTIN. }\end{array}$ \\
\hline $\begin{array}{l}\text { Lorenzini et al., } \\
2013 \\
\text { Revista Gaúcha } \\
\text { de Enfermagem }\end{array}$ & $\begin{array}{l}\text { Prevenção e controle } \\
\text { de infeção em } \\
\text { unidade de terapia } \\
\text { intensiva neonatal }\end{array}$ & $\begin{array}{l}\text { Identificar o conhecimento da } \\
\text { equipe de enfermagem de uma } \\
\text { Unidade de Terapia Intensiva } \\
\text { Neonatal sobre o controle de } \\
\text { infecção, identificando os } \\
\text { fatores que facilitam ou } \\
\text { dificultam o controle e } \\
\text { prevenção das Infecções } \\
\text { Relacionadas à Assistência à } \\
\text { Saúde (IRAS). }\end{array}$ \\
\hline $\begin{array}{l}\text { Tomaz et al., } \\
2011 \\
\text { Revista da } \\
\text { Rede de } \\
\text { Enfermagem do } \\
\text { Nordeste }\end{array}$ & $\begin{array}{l}\text { Medidas de prevenção } \\
\text { e controle de infecções } \\
\text { neonatais: opinião da } \\
\text { equipe de enfermagem }\end{array}$ & $\begin{array}{l}\text { Conhecer a opinião da equipe } \\
\text { de enfermagem sobre as } \\
\text { medidas de prevenção e } \\
\text { controle das infecções } \\
\text { neonatais nos recém-nascidos } \\
\text { na unidade neonatal. }\end{array}$ \\
\hline $\begin{array}{l}\text { Pettit \& Sharpe, } \\
2017 \\
\text { Neonatal } \\
\text { network }\end{array}$ & $\begin{array}{l}\text { The effect of } \\
\text { education on } \\
\text { hlorhexidine use in the } \\
\text { NICU }\end{array}$ & $\begin{array}{l}\text { Determinar o efeito de um } \\
\text { programa de educação } \\
\text { direcionado sobre o } \\
\text { conhecimento e as atitudes dos } \\
\text { enfermeiros que colocam } \\
\text { cateteres centrais inseridos } \\
\text { perifericamente na UTIN em } \\
\text { relação ao uso e remoção do } \\
\text { anti-séptico gluconato de } \\
\text { clorexidina (CHg). }\end{array}$ \\
\hline $\begin{array}{l}\text { Cebalos et al., } \\
2013 \\
\text { Advances in } \\
\text { Neonatal Care }\end{array}$ & $\begin{array}{l}\text { Nurse-driven quality } \\
\text { improvement } \\
\text { interventions to reduce } \\
\text { hospital-acquired } \\
\text { infection in the NICU }\end{array}$ & $\begin{array}{l}\text { Aplicar as intervenções dos } \\
\text { pacotes de prevenção } \\
\text { específicos da UTIN visando } \\
\text { eliminar infecção da corrente } \\
\text { sanguínea relacionado ao } \\
\text { cateter venoso central (ICSRC) } \\
\text { e pneumonia associada a } \\
\text { ventilação mecânica (PAV) de } \\
\text { maneira sistemática e, ao longo } \\
\text { do tempo, medir a eficácia nos } \\
\text { resultados dos pacientes em } \\
\text { todas as } 5 \text { categorias de peso } \\
\text { ao nascer de neonatos. }\end{array}$ \\
\hline $\begin{array}{l}\text { Taylor et al., } \\
2011 \\
\text { Advances in } \\
\text { Neonatal Care }\end{array}$ & $\begin{array}{l}\text { Effect of a dedicated } \\
\text { percutaneously } \\
\text { inserted central } \\
\text { catheter team on } \\
\text { neonatal catheter- } \\
\text { related bloodstream } \\
\text { infection }\end{array}$ & $\begin{array}{l}\text { Avaliar se o estabelecimento } \\
\text { de uma equipe dedicada de } \\
\text { cateter central inserido } \\
\text { percutaneamente (PICC) está } \\
\text { associada a risco reduzido de } \\
\text { infecção da corrente sanguínea } \\
\text { relacionado ao cateter (ICSRC) } \\
\text { na unidade de terapia intensiva } \\
\text { neonatal. }\end{array}$ \\
\hline $\begin{array}{l}\text { Costa et al., } \\
2013 \\
\text { Journal of } \\
\text { Obstetric, } \\
\text { Gynecologic \& } \\
\text { Neonatal } \\
\text { Nursing }\end{array}$ & $\begin{array}{l}\text { Incidence of } \\
\text { nonelective removal } \\
\text { of percutaneously } \\
\text { inserted central } \\
\text { catheters according to } \\
\text { tip position in } \\
\text { neonates }\end{array}$ & $\begin{array}{l}\text { Comparar a incidência e as } \\
\text { razões para a remoção não } \\
\text { seletiva de cateteres centrais } \\
\text { inseridos percutaneamente } \\
\text { (linhas PICC) entre linhas } \\
\text { PICC colocadas central e não } \\
\text { centralmente em neonatos. }\end{array}$ \\
\hline
\end{tabular}




\begin{tabular}{|c|c|c|}
\hline $\begin{array}{l}\text { Balegar et al., } \\
2013 \\
\text { Journal of } \\
\text { Paediatrics and } \\
\text { Child Health }\end{array}$ & $\begin{array}{l}\text { Extending total } \\
\text { parenteral nutrition } \\
\text { hang time in the } \\
\text { neonatal intensive care } \\
\text { unit: Is it safe and cost } \\
\text { effective? }\end{array}$ & $\begin{array}{l}\text { Investigar os efeitos do } \\
\text { prolongamento do tempo de } \\
\text { espera do fluido de nutrição } \\
\text { parenteral total (NPT) na } \\
\text { infecção da corrente sanguínea } \\
\text { relacionado ao cateter venoso } \\
\text { central (ICSRC), custo } \\
\text { relacionado à NPT e carga de } \\
\text { trabalho de enfermagem. }\end{array}$ \\
\hline $\begin{array}{l}\text { Moraes et al., } \\
2019 \\
\text { Revista da } \\
\text { Escola de } \\
\text { Enfermagem da } \\
\text { USP }\end{array}$ & $\begin{array}{l}\text { Assistência de } \\
\text { enfermagem ao } \\
\text { recém-nascido com } \\
\text { Ictiose Lamelar: um } \\
\text { estudo de caso em } \\
\text { unidade neonatal }\end{array}$ & $\begin{array}{l}\text { Apresentar os cuidados de } \\
\text { enfermagem de um recém- } \\
\text { nascido com Ictiose Lamelar } \\
\text { internado em unidade de } \\
\text { terapia intensiva neonatal de } \\
\text { um hospital público infantil, } \\
\text { em município do estado do } \\
\text { Paraná. }\end{array}$ \\
\hline $\begin{array}{l}\text { Raines et al., } \\
2017 \\
\text { Neonatal } \\
\text { network }\end{array}$ & $\begin{array}{l}\text { Intraamniotic infection } \\
\text { and the term neonate }\end{array}$ & $\begin{array}{l}\text { Descrever a fisiopatologia da } \\
\text { infecção intra-amniótica e o } \\
\text { papel da enfermeira mãe-bebê } \\
\text { durante o nascimento } \\
\text { antecipado e a avaliação } \\
\text { contínua do recém-nascido. }\end{array}$ \\
\hline $\begin{array}{l}\text { Mangoejane et } \\
\text { al., } 2019 \\
\text { African Journal } \\
\text { of Primary } \\
\text { Health Care \& } \\
\text { Family } \\
\text { Medicine }\end{array}$ & $\begin{array}{l}\text { Implementation of } \\
\text { postnatal care for } \\
\text { HIV-positive mothers } \\
\text { in the Free State: } \\
\text { Nurses' perspectives }\end{array}$ & $\begin{array}{l}\text { Explorar a implementação do } \\
\text { cuidado pós-natal para } \\
\text { mulheres soropositivas, } \\
\text { explicando a visão das } \\
\text { enfermeiras sobre suas } \\
\text { práticas. }\end{array}$ \\
\hline $\begin{array}{l}\text { Pletsch et al., } \\
2013 \\
\text { Nursing } \\
\text { leadership }\end{array}$ & $\begin{array}{l}\text { Mothers' "liquid } \\
\text { gold": A quality } \\
\text { improvement initiative } \\
\text { to support early } \\
\text { colostrum delivery via } \\
\text { oral imune therapy } \\
\text { (OIT) to premature } \\
\text { and critically ill } \\
\text { newborns }\end{array}$ & $\begin{array}{l}\text { Facilitar a disponibilidade da } \\
\text { imunoterapia oral } \\
\text { (fornecimento de colostro) } \\
\text { para neonatos prematuros e } \\
\text { gravemente enfermos o mais } \\
\text { rápido possível após o } \\
\text { nascimento. }\end{array}$ \\
\hline $\begin{array}{l}\text { Williams et al., } \\
2013 \\
\text { Journal of } \\
\text { Obstetric, } \\
\text { Gynecologic \& } \\
\text { Neonatal } \\
\text { Nursing }\end{array}$ & $\begin{array}{l}\text { Influenza infection } \\
\text { control practices in } \\
\text { labor and delivery } \\
\text { units during the } 2009 \\
\text { H1N1 influenza } \\
\text { pandemic }\end{array}$ & $\begin{array}{l}\text { Avaliar a presença e utilidade } \\
\text { de políticas e práticas escritas } \\
\text { sobre controle de infecção } \\
\text { consistentes com a orientação } \\
\text { do Centro de Controle e } \\
\text { Prevenção de Doenças (CDC) } \\
\text { em unidades de parto } \\
\text { hospitalar (L\&D) durante a } \\
\text { pandemia de influenza H1N1 } \\
\text { de } 2009 \text {. }\end{array}$ \\
\hline $\begin{array}{l}\text { Zapata et al., } \\
2013 \\
\text { Nursing for } \\
\text { women's health }\end{array}$ & $\begin{array}{l}\text { Postpartum and } \\
\text { neonatal nursing care } \\
\text { during the } 2009 \mathrm{H} 1 \mathrm{~N} 1 \\
\text { influenza pandemic }\end{array}$ & $\begin{array}{l}\text { Compreender o grau em que as } \\
\text { práticas recomendadas pelo } \\
\text { Centro de Controle e } \\
\text { Prevenção de Doenças (CDC) } \\
\text { foram apoiadas e } \\
\text { implementadas em hospitais } \\
\text { durante e após a pandemia, e } \\
\text { para examinar o nível de } \\
\text { dificuldade que as enfermeiras } \\
\text { experimentaram na } \\
\text { implementação de certas } \\
\text { práticas. }\end{array}$ \\
\hline
\end{tabular}

Fonte: os autores

\section{DISCUSSÃO}

Por meio da análise dos resultados, foi possível verificar aspectos importantes relacionados às ações da equipe de enfermagem para prevenção e controle de infecção neonatal. As ações prestadas ao controle de infecções neonatais em áreas hospitalares são de suma importância onde o contato entre pacientes e funcionários da saúde seja corriqueiro nessas situações. Assim, infere-se que as infecções do período neonatal contemplam tanto as infecções relacionadas à assistência (IRAS) quanto as relacionadas a falhas nas medidas de prevenção, diagnóstico e tratamento ${ }^{10}$.

Há formas de prevenção na questão do cuidado ao neonato como a lavagem das mãos e uso de equipamentos de proteção individual como luvas, capote e óculos. Essas ações evitam a disseminação de microrganismos por meio do contato direto entre profissional de saúde e neonato, como por exemplo no momento de administrar medicamentos, preparar a nebulização ou ter um contato com o recémnascido ${ }^{11}$. Assim, ter um controle sobre a realização dos procedimentos de forma asséptica constitui fator básico de proteção para o bloqueio da disseminação de patógenos no âmbito das unidades de terapias neonatais ${ }^{10,11}$.

Ressalta-se que os cuidados de enfermagem devem ser pautados na humanização da assistência, levando em consideração o recém-nascido, a mãe e sua família. A equipe de enfermagem deve estar sempre atenta aos sinais vitais, administração de medicamentos, restrição de visitas, orientação para a mãe e para a família sobre noções de assepsia e mudanças do quadro clínico do neonato. Além disso, cabe ao enfermeiro neonatologista avaliar o recémnascido, supervisionar a equipe de enfermagem nos cuidados de higiene, tanto da equipe quanto dos pais, na limpeza da unidade e no controle dos horários de visita ${ }^{10,11}$.

Ao se depararem com as IRAS, os enfermeiros precisam estar atentos principalmente nos cuidados relacionados à pele do recém-nascido, pois, nesse momento a pele do bebê torna-se principal rota para infecções. Procedimentos invasivos, tempo prolongado de internação, baixo peso ao nascimento e o contato precoce com os pais sem a devida assepsia são fatores que podem desencadear um aumento das IRAS ${ }^{12}$.

Nesse contexto, o uso de antissépticos na pele é um procedimento importante para a implantação de cateteres centrais dentro das UTIN $^{13}$. Estudos revelaram que a utilização do antisséptico gluconato de clorexidina $(\mathrm{CHg})$ durante e após a inserção de um cateter central foi benéfico na prevenção de infecções neonatais ${ }^{14}$. Outro estudo apontou que a utilização do ChloraPrep (CHg 2\% / álcool isopropílico $70 \%$ ) ou iodopovidona para a antissepsia da pele de neonato 
reduziram a incidência de infecções associadas ao cateter central $^{15}$

Outra ação importante para a redução dos índices de infecção nas UTI's neonatais foi a avaliação das inserções e manejo da retirada dos cateteres de inserção periférica (PICC) pela equipe de enfermagem. Pesquisas verificaram que o manuseio incorreto e a remoção não seletiva dos cateteres (sem avaliação de sinais flogísticos) foram possíveis desencadeantes de infecções neonatais ${ }^{16,17}$.

Outra variável investigada quanto ao risco de infecção associada ao cateter central foi o tempo de permanência do dispositivo para nutrição parenteral total (NPT). Estender o tempo de espera da NPT de 24 horas com bolsa de $500 \mathrm{ml}$ para 48 horas com bolsa de $1000 \mathrm{ml}$ não alterou a taxa de infecção associada ao cateter central. Além disso, o prolongamento do período do cateter central para a NPT reduziu os custos, o tempo gasto na troca do circuito da NPT e a carga de trabalho de enfermagem ${ }^{18}$.

Frente ao exposto, a Sistematização da Assistência de Enfermagem no âmbito da UTI neonatal garante uma assistência integral e humanizada ao binômio mãe-filho, além de garantir ações que promovam a prevenção de infecções neonatais. Assim, os cuidados de enfermagem que podem estar envolvidos na prevenção do risco de infecção à neonatos estão: manter permeabilidade dos acessos venosos centrais e periféricos; utilizar técnica asséptica na administração de medicamentos; trocar extensores e torneirinhas a cada 72 horas; avaliar hidratação de mucosas; higienizar os olhos do RN com solução hialina; aplicar colírio lubrificante sempre que necessário; administrar Acitretina, conforme prescrição médica para casos de ressecamento ocular pela deficiência de vitamina $\mathrm{A}$; hidratar lábios com vaselina antes da administração da dieta oral, dentre outros ${ }^{19}$.

Outra importante intervenção de enfermagem na prevenção e controle de infeções neonatais é a identificação precoce de sepse. Fetos a termo geralmente se apresentam infecções entre as 6 e 24 horas após o parto. Portanto, é importante que o enfermeiro reconheça sinais e sintomas precoces da sepse neonatal, tais como a diminuição da atividade espontânea, uma sucção menos vigorosa, anorexia, apneia, bradicardia e instabilidade térmica (hipo ou hipertermia). Assim, a avaliação abrangente e a identificação precoce dos sintomas são fatores-chave no fornecimento de tratamento terapêutico oportuno ${ }^{20}$.

Observou-se que os enfermeiros também avaliaram o processo da amamentação para a prevenção de infecções. Práticas educativas em saúde foram realizadas que enfatizaram o autocuidado das mamas da mãe, no intuito de verificar a presença de sinais de infecções durante a ordenha do leite materno. Além disso, os enfermeiros promoveram ações para a obtenção de colostro materno para a imunoterapia oral precoce de neonatos que não poderiam ser amamentados diretamente, visando a redução do risco de infecção nosocomial e enterocolite necrosante ${ }^{21,22}$.

Em um contexto de pandemia de H1N1, em 2009, enfermeiras neonatais implantaram políticas de prevenção à infecção neonatal, tais como o isolamento de mães com suspeita ou infectadas pelo $\mathrm{H} 1 \mathrm{~N} 1$; os próprios enfermeiros foram responsáveis pela higiene e alimentação dos neonatos ${ }^{23}$. Estas informações aprendidas podem ser úteis para orientar o planejamento de práticas para proteger neonatos imunologicamente imaturos durante futuras pandemias como o Covid-19 ou para outras emergências de saúde pública ${ }^{24}$.

\section{CONCLUSÃO}

Esta revisão destaca aspectos importantes durante os cuidados de enfermagem para a prevenção e controle de infecções em neonatos, contribuindo para o conhecimento de enfermeiros e demais profissionais de saúde.

$\mathrm{O}$ rigor das medidas estabelecidas para a segurança e qualidade do cuidado é essencial para a redução da mortalidade neonatal. Neste trabalho foi possível verificar diferentes ações realizadas por enfermeiros para o controle de infecções, destacando-se a lavagem das mãos, a utilização de proteção pessoal como luvas, capote e óculos, os cuidados com a pele, a utilização de antissépticos para a implantação de cateteres centrais e orientações relacionadas à amamentação e os cuidados envolvendo a participação dos pais.

Ressalta-se que os enfermeiros são o elo entre a equipe de enfermagem e os demais profissionais da saúde que atuam na assistência ao neonato de forma direta ou indireta. Os enfermeiros neonatais realizam intervenções de alta complexidade para a manutenção da vida, além de atuar na prevenção e promoção à saúde, com trabalhos voltados para o esclarecimento dos pais quanto aos cuidados do neonato.

A capacitação e o aperfeiçoamento destes profissionais são necessários para suas práticas de excelência. Assim, é importante que trabalhos educativos sejam realizados continuamente com a finalidade de atualizar, esclarecer, reforçar e melhorar a qualidade, segurança e eficácia do cuidado de enfermagem.

Ressalta-se a necessidade de novas pesquisas no âmbito da neonatologia e da enfermagem neonatológica a fim de garantir práticas baseadas em evidência científica e 
garantir uma assistência livre de infecções neonatais que podem ser evitadas.

\section{REFERÊNCIAS}

1. World Health Organization (WHO). Newborns: improving survival and well-being [Internet]. 2020 [acessado em 08 jun. 2021]; Disponível em: https://www.who.int/news-room/fact-sheets/detail/newborns-reducingmortality

2. Ministério da Saúde (BR). Óbitos por causas evitáveis em menores de 5 anos - Brasil. 2019 [acessado em 08 jun. 2021]; Disponível em: http://tabnet.datasus.gov.br/cgi/deftohtm.exe?sim/cnv/evita10uf.def

3. Ministério da Saúde (BR), Agência Nacional de Vigilância Sanitária (ANVISA). Neonatologia: Critérios nacionais de infecção relacionados à assistência à saúde. Brasília, DF, 2008 [acessado em 08 jun. 2021]; Disponível em:

https://www.anvisa.gov.br/servicosaude/manuais/manual definicao criterios n acionais infec\%E7\%F5es relacionadas assistencia saude neonatologia.pdf

4. Association of Women's Health, Obstetric and Neonatal Nurses (AWHONN). Neonatal Nursing: Clinical Competencies and Education Guide, 7th edition. Nurs Womens Health [Internet]. 2019 [acessado em 18 jun. 2021]; 23(3):e23-e35. Doi; https://doi.org/10.1016/j.nwh.2019.04.002

5. Kaye A. Toxoplasmosis: diagnosis, treatment, and prevention in congenitally exposed infants. J Pediatr Health Care [Internet]. 2011 [acessado em em 25 jun. 2021]; 25(6):355-364. Doi:

https://doi.org/10.1016/j.pedhc.2010.04.008

6. Ruch-Ross HS, Zapata LB, Williams JL, Ruhl C. General influenza infection control policies and practices during the 2009 H1N1 influenza pandemic: A survey of women's health, obstetric, and neonatal nurses. Am J Infect Control [Internet]. 2014 [acessado em em 08 mar. 2021]; 42(6):e65-e70. Disponível em:

https://www.ncbi.nlm.nih.gov/pmc/articles/PMC4322902/pdf/nihms655988.pd $\underline{\mathrm{f}}$

7. Mutic AD, Jordan S, Edwards SM, Ferranti EP et al. The Postpartum Maternal and Newborn Microbiomes. MCN Am J Matern Child Nurs [Internet]. 2017 [acessado em 08 mar. 2021]; 42(6):326-331. Disponível em: https://www.ncbi.nlm.nih.gov/pmc/articles/PMC5649366/pdf/nihms848770.pd

8. Rowe CR, Newberry DM, Jnah AJ. Congenital Syphilis: A Discussion of Epidemiology, Diagnosis, Management, and Nurses' Role in Early Identification and Treatment. Adv Neonatal Care [Internet]. 2018 [acessado em 30 jun. 2021]; 18(6):438-445. Doi:

https://doi.org/10.1097/anc.0000000000000534

9. Torraco JR. Writing integrative literature reviews: guidelines and examples. Human Resource Development Review [Internet]. 2005 [acessado em 08 mar. 2021]; 4(3):356-367. Disponível em:

https://journals.sagepub.com/doi/pdf/10.1177/1534484305278283

10. Cunha KJB, Moura MEB, Nery IS, Rocha SS. Representações sociais de infecção neonatal elaboradas por enfermeiras. Rev enferm UERJ [Internet]. 2013 [acessado em 08 mar. 2021]; 21(4):527-532. Disponível em: file://C:/Users/bezer/Downloads/10028-34711-1-PB\%20(1).pdf

11. Hoogen AVD, Brouwer AJ, Verboon-Maciolek MA. Improvement of adherence to hand hygiene practice using a multimodal intervention program in a neonatal intensive care. J Nurs Care Qual [Internet]. 2011 [acessado em 25 jun. 2021]; 26(1):22-29. Doi: https://doi.org/10.1097/ncq.0b013e3181ea86e9

12. Lorenzini E, Costa TC, Silva EF. Prevenção e controle de infecção em unidade de terapia intensiva neonatal. Rev Gaúcha Enferm [Internet]. 2013 [acessado em 08 mar. 2021]; 34(4):107-113. Disponível em:

https://www.scielo.br/pdf/rgenf/v34n4/en_14.pdf
13. Tomaz VS, Campos-Neto FH, Almeida PC, Maia RCF et al. Medidas de prevenção e controle de infecções neonatais: opinião da equipe de enfermagem. Rev Rene [Internet]. 2011 [acessado em 08 mar. 2021]; 12(2):271-278. Disponível em: https://www.redalyc.org/pdf/3240/324027975004.pdf

14.Pettit JD \& Sharpe EL. The effect of education on hlorhexidine use in the NICU. Neonatal Netw [Internet]. 2017 [acessado em 25 jun. 2021]; 36(5):294305. Doi: https://doi.org/10.1891/0730-0832.36.5.294

15. Ceballos K, Waterman K, Hulett T, Makic MBF. Nurse-driven quality improvement interventions to reduce hospital-acquired infection in the NICU. Adv Neonatal Care [Internet]. 2013 [acessado em 28 jun. 2021]; 13(3):154-63. Doi: https://doi.org/10.1097/anc.0b013e318285fe70

16. Taylor T, Massaro A, Williams L, Doering J et al. Effect of a dedicated percutaneously inserted central catheter team on neonatal catheter-related bloodstream infection. Adv Neonatal Care [Internet]. 2011 [acessado em 27 jun. 2021]; 11(2):122-128. Doi: https://doi.org/10.1097/anc.0b013e318210d059

17. Costa P, Bueno M, Alves AMA, Kimura AF. Incidence of nonelective removal of percutaneously inserted central catheters according to tip position in neonates. J Obstet Gynecol Neonatal Nurs [Internet]. 2013 [acessado em 30 mai. 2021]; 42(3):348-56. Doi: https://doi.org/10.1111/1552-6909.12030

18. Balegar-V KK, Azeem MI, Spence K, Badawi N. Extending total parenteral nutrition hang time in the neonatal intensive care unit: Is it safe and cost effective? J Paediatr Child Health [Internet]. 2013 [acessado em 25 jun. 2021]; 49(1):E57-61. Doi: https://doi.org/10.1111/jpc.12023

19. Moraes ELL, Freire MHS, Rocha F, Secco IL et al. Assistência de enfermagem ao recém-nascido com Ictiose Lamelar: um estudo de caso em unidade neonatal. Rev esc enferm USP [Internet]. 2019 [acessado em 08 mar. 2021]; 53:e03519. Disponível em: https://www.scielo.br/pdf/reeusp/v53/1980220X-reeusp-53-e03519.pdf

20. Raines DA, Wagner A, Salinas A. Intraamniotic infection and the term neonate. Neonatal Netw [Internet]. 2017 [acessado em 27 jun. 2021]; 36(6):385-387. Disponível em

https://connect.springerpub.com/content/sgrnn/36/6/385

21 Mangoejane L \& Ramukumba MM. Implementation of postnatal care for HIV-positive mothers in the Free State: Nurses' perspectives. Afr J Prim Health Care Fam Med [Internet]. 2019 [acessado em 08 mar. 2021]; 11(1):e1 e8. Disponível em:

https://www.ncbi.nlm.nih.gov/pmc/articles/PMC6489183/pdf/PHCFM-111776.pdf

22. Pletsch D, Ulrich C, Angelini M, Fernandes G et al. Mothers" "liquid gold": A quality improvement initiative to support early colostrum delivery via oral imune therapy (OIT) to premature and critically ill newborns. Nurs.

Leadersh (Tor. Ont) [Internet]. 2013 [acessado em 08 mar. 2021]; 26 Spec No 2013:34-42. Disponível em:

https://www.longwoods.com/content/23356/nursing-leadership/mothers-liquidgold-a-quality-improvement-initiative-to-support-early-colostrum-delivery-viaora

23. Williams JL, Mersereau PW, Ruch-Ross H, Zapata LB et al. Influenza infection control practices in labor and delivery units during the $2009 \mathrm{H} 1 \mathrm{~N}$ influenza pandemic. J Obstet Gynecol Neonatal Nurs [Internet]. 2013 [acessado em 08 mar. 2021]; 42(5):527-540. Disponível em: https://www.ncbi.nlm.nih.gov/pmc/articles/PMC4447205/pdf/nihms692527.pd $\underline{f}$

24. Zapata LB, Ruch-Ross HS, Williams JL, Ruhl C. Postpartum and neonatal nursing care during the $2009 \mathrm{H} 1 \mathrm{~N} 1$ influenza pandemic. Nurs Womens Health [Internet]. 2013 [acessado em 08 mar. 2021]; 17(4):284-293. Disponível em: https://www.ncbi.nlm.nih.gov/pmc/articles/PMC5061496/pdf/nihms818376.pd 